\title{
Collaborative Design Squared Creating together the tools for working together
}

\author{
Rafael Lemieszek Pinheiro \\ Escola de Arquitetura da UFMG, Brazil \\ lemieszek@gmail.com
}

\begin{abstract}
This article discusses the use of collaborative processes in architecture and urban design, based on an experience of collaborative design that took place in UFOP, Brazil, in which different digital interfaces were used to create a preliminary design for a new building in the university campus. The process is described here in detail. This paper concludes that, since the way each collaborative process is designed heavily influences its output, the process should, itself, be designed collaboratively.
\end{abstract}

Keywords: Collaborative Platforms; Collaborative Design; Parametric Tools; Collective Process; Design Process.

\section{Introduction}

This article discusses the use of collaborative processes in architecture and urban design, based on an experience of collaborative design that took place in Universidade Federal de Ouro Preto (UFOP), located in the city of Ouro Preto, Brazil. In this experience, different digital interfaces were used to create a preliminary design for a new building in the university campus.

The first part of the article (Methodology and Results) describes how each platform was used to gauge general and specific demands that the academic community had for this new building, analyzing how different interfaces influenced the input that each stakeholder was able to express towards the building program. The second part (Collective Metadesign Processes) focuses on the actual design process, which took place predominantly using Grasshopper (a parametric plugin for Rhinoceros, a 3D modeling tool) and a few of its free addons. Each participant was able to download, edit and upload any working version of the design, which started with diagrams and ended up with a schematic model of the proposed design. There were two dimensions of participation in this process: first, each member of the academic community was able to contribute to the design in whichever way they were comfortable with at each design iteration, according to their level of expertise in architecture (ranging from "none", i.e., students from unrelated courses, to "specialist", i.e. graduated architects). Second, each new design iteration brought about new information over which each of the stakeholders was able to act upon according to their abilities and expectations.

The final part of the article argues that advancing collaborative design relies on understanding and advancing two gradients of participation that are closely related to the concept of second-order cybernetics. First, they must be able to absorb contributions from people with different levels of expertise, since these processes usually include very different stakeholders, and setting a precondition that all stakeholders must possess similar levels of knowledge greatly limits the purpose of collaborative processes. To that end, visual interfaces are paramount to allow people with different levels of expertise to understand and to act upon the design process that is being carried out. The second gradient is the process by which each new collaborative experience advances each individual's ability to take part in them, so that each specific collaborative process is improved by previous experiences and, in turn, improves the following ones for everyone involved.

\section{Methodology and Results Context and Preliminary Considerations}

The experiment hereby described was inspired by a longstanding but increasing trend of exploring collective processes for designing collective and public spaces and buildings. The increasingly specialized profession of architecture and urban planning and the growing complexity of the built space has been widening the gap between processed knowledge (from technocrats) and personal experience (from citizens/users), and this gap has been the subject of many different texts since at least the 1970s (Friedmann, 1987).

Despite a widely recognized need for the increase of "smart citizenship", or citizen participation in political processes (Noveck, 2015), the body of knowledge on actual practices aimed at bridging the gap between specialized knowledge and personal experience is, so far, somewhat thin. This seems to be at least in part due to what Kapp and Baltazar (2012) have called the Paradox of Participation: the idea that the process of taking part in something is usually mediated by institutional structures that allow for it and, by doing so, restrict or dictate when, where and to what degree stakeholders are able to take part. In other words, being a part of a collaborative process is of little effectiveness when the rules that mediate relationships in that process are predetermined by someone else.

On the other hand, processes that intend to determine the design of a building or a public space need legitimacy. The paradox is, therefore, this: the process needs to be structured to take place and needs to be publicly available for all to be 
legitimate, but the way it is structured can play an equal or greater part in its outcome than the actual actions of its participants. The only way to avoid this is to open up not just the design process, but also the platforms over which people collaborate and, furthermore, the rules of the interactions that take place on those platforms.

However, while a predesigned structure for collaboration might be paradoxical, moving too much towards the opposite end of the spectrum risks stalling any initiative that might catalyze a collaborative process. In other words, a minimal amount of institutionalized or structured action must happen at some point for any collaborative action to take place. This can happen without delegitimizing the entire process, as long as the initial efforts are seen as provisional and hand over the power of deciding and taking any actions to the participants in the process - including changing the rules of interaction or even the option of scraping the initiative altogether.

\section{A means - not just to an end, but to further means}

What this means is that the only way for any collaborative effort to avoid becoming paradoxical is if it allows itself to be changed by its participants not only in its results, but also in its processes. However, as stated, the way in which people interact depends on the rules and platforms over which they do so. There is, thus, a cyclical dependence between collaborative platforms and collaborating individuals; this is the very definition of a cybernetic relationship. It might be said, then, that a collaborative process is an effective way of desimplifying architecture where it needs to, since, as per W. R. Ashby's Law of Requisite Variety (1964), a controlled system (in this case, the design of a building) can only have as many possible states as the controller system (in this case, the collaborative platform).

Most design processes must, however, reach an execution phase at some point. Even if a building or a public space remains open to further changes and interactions, there must be a point where a conceptual design is deemed concluded, regardless of how provisional that conclusion is, and advance to a construction phase. There is a risk, then, that prospective stakeholders in a given collaborative process might find it too much of a hassle to get together and discuss not only design ideas, but also platforms of interaction, rules of discussion and so on.

In that context, the gain from new ideas emerging from collaborative processes might be seen as outweighed by the energy that has to be put in forming and promoting the means through which people of different levels of expertise and involvement might interact and agree upon. If the only thing that matters is the product of that process - in this case, a finished building -, then it might be justifiable for a community to delegate power to an individual or a group that they know to be competent in architectural design. This is far from unacceptable and is probably justifiable in several cases. Nevertheless, the cybernetic relationship between users and platforms means that every new experience enhances an individual's abilities to take part in the next one, and that every new, provisional collaborative platform might serve as the basis for the next one.

It is not by chance that Sherry Arnstein's proposed way of enhancing citizen participation is illustrated with something as inherently incremental as a ladder ("A Ladder of Citizen Participation" (Arnstein, 1969)), and not something more seductive but definitive. In that sense, providing ways for people to interact with something as trivial as the built environment in which they live, work or play might be both an easier and a more effective first step towards direct democracy than complex national legislation that aim at quickly and definitively changing the participation state-of-the-art.

This spirit has animated the experience hereby described. The exercise of designing new infrastructure for the architecture course at the Federal University of Ouro Preto (UFOP) was understood to be an adequate opportunity for exercising and advancing some of these concepts. Once the design was done, publishing the experience to help inspire and structure further experiences elsewhere is understood as the way to move the participation state-of-the-art forward.

The following section will describe the adopted process, as well as the successes, failures and improvements proposed along the way.

\section{Collective Metadesign Process}

Two provisional first steps were taken to gather students from the architecture school around the subject and kickstart the process. One, the definition that a workshop would take place in the two first months of the semester, with two weekly meetings of three hours each, which is equivalent to a 60-hour course. Two, that due to the dynamic nature that the collaborative process was expected to assume, with each part of it being understood as provisional until everyone was satisfied with the result, the workshop would be themed around "parametric tools geared towards collaboration". The length of the workshop, as well as the actual tools to be used in it, were left open to being adjusted by the participants later on.

A total of 88 architecture students signed up for the workshop. Due to physical limitations of the space where it would take place - and one of the instances in which a heteronomous, if provisional, decision had to be made in the name of practicality - a limit of 25 students was set. An e-mail message was sent to students who had signed up explaining this and proposing that, if anyone had different expectations of what the workshop would be, they could take part in it as external agents keeping track of what the group was doing through the internet and helping whenever and however they felt they could. Any student still interested in taking part in the meetings was invited to send another e-mail confirming their interest. 59 students opted to take an external role and 29 confirmed their interest in the workshop, which was deemed acceptable.

\section{Defining the problem}

Some of the context presented above was discussed in the first meeting. The students were then invited to start thinking about 
what the group would be working on in the next few weeks. Some students inquired what kind of project we would be developing together. This, too, was left open for discussion. We identified that there was an initial resistance on everybody's part to escape the life-long psychological programming of a normal professor-student relationship. The way the classroom dedicated to the workshop was designed, with fixed tables turned towards an elevated platform on one end of the room, was identified as both a consequence and a cause for this. We decided to place the chairs forming a circle, as much as the room would allow, to break some of the initial resistance.

With this initial resistance came the realization that the relationship between the different stakeholders in the process could not be expected to be perfectly horizontal. If, on the one hand, participatory process are conditioned upon opening up the black box of "the secret profession of architecture" (Banham, 1991), on the other, it is reasonable to expect that people with different knowledges, needs and backgrounds can (and want to) participate in different ways. With this in mind, the group laid out a conceptual diagram of the different kinds of participants that the collaborative process could be expected to involve (Figure 1).

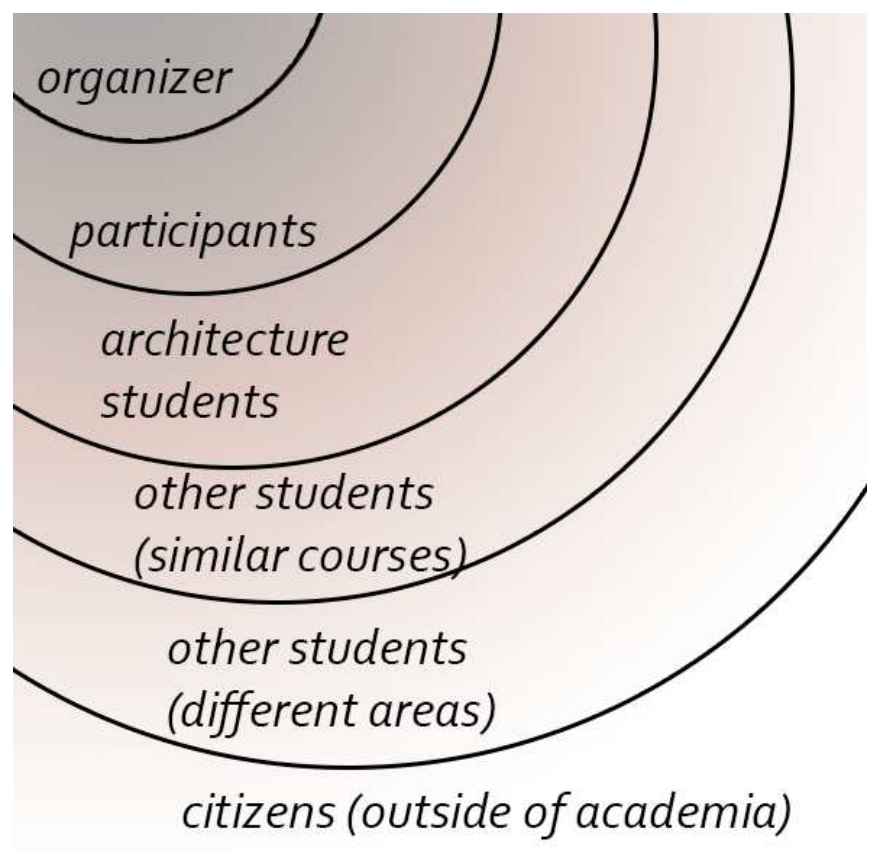

Figure 1 - Gradient of the different expected participants in the collaborative process proposed. Recreated from a drawing on the board.

The diagram was not intended to delineate hierarchy and is not expected to be exhaustive, but to give visibility to the idea that different types of users naturally feel more or less involved in conceiving the new building, both for their expected frequency of the area and for their previous knowledge about the matter. Each new circle indicates a group that is an order of magnitude larger in number, but less naturally involved in the process.
Two principles were then proposed: one, that there were no decisions that were off-limits to anyone in or outside the group; and two, that any decision taken during any part of the process was to be understood as provisional until the process was deemed concluded by the group.

The first problem to be tackled was, then, deciding on what platforms would be able to bring together all different layers of potential collaborators in the process. Different online platforms were analyzed. One in particular, called Loomio, was considered to be convenient for offering different types of interaction (discussion forums, different voting interfaces and so on). However, its learning curve was seen as steep to some of the participants, who felt that could be an obstacle for widespread participation. The group then ultimately decided to stick to two very popular platforms with which the academic community is already comfortable: Facebook and Google Drive.

Two groups were created on Facebook: one for participants in the workshop, for internal discussions and day-to-day communication, and another open for anyone to join. In the following week, 245 members of the academic community had joined the open group.

The second decision to be made regarded exactly what kind of building (if indeed a building) would be proposed. The group identified the need for a pavilion to support the architecture course in different activities, but also recognized that, while located in a campus with several different courses, all academic community should have a say in what was to be proposed. The group decided that it would be a good idea do spread posters around the university campus over which anyone could write or draw anything. As a way help people getting used to the decision making processes, the group decided that the posters would carry a motto - a catchphrase to invite people to interact with it. With the intention of helping people get used to voicing opinions and taking part in the decision-making processes, a poll was launched in the open group to gather ideas about what that motto might be. People could vote on one of the options initially laid out or they could write-in new suggestions. A date was set for the voting to close, and the phrase "Faça Seu Campus" ("Make Your Campus") won.

The design of the posters was then seen as an interesting first step towards investigating collaborative design processes. Any member of the open group could submit ideas for the layouts, and, again, a voting took place to decide which one would be used. One layout template was chosen, and three types of posters were drawn: one completely open, which just the motto on top; one with a question as subtitle ("What would you like to see built or improved on the campus?"); and one with an axonometric view of a land plot, with threes and people for scale, and the phrase "Draw a new building for the campus". The idea was to test the different kinds of interactions that each type of poster would yield. Twelve posters were printed and posted around the campus.

All three types of posters showed interactions that the group later separated in three types: one that was irrelevant to the 
process (jokes, abstract sketches, etc.); one that was seen as indirectly relevant (phrases such as "people should interact with each other more", or "we need a place where we feel welcome"); and one seen as directly relevant ("we need bike parking spots" or "we need places where we can sit and work together"). As expected, the "open" poster layout yielded more of the former and less of the latter, while the "draw a new building" layout produced more applied interactions.

\section{Deciding on a program}

Once the different types of users were made aware of the process and began engaging in it, the group decided on a framework for the actual design. Two different documents were created, edited together among the internal group and then made available to everyone else in the community: the first one was a shared Google Document called Dynamic Guidelines Document, intended as a provisional walk-through of the entire design process that was to be updated by anyone at any time. It contained a general introduction in the first page and a series of five steps that were to be taken along the way, each of them with two subsections: "General Objectives" and "Specific Actions". Each step was to be followed by a general discussion about it and a revisit to the preceding steps to see if anyone felt like changing anything in the document for future reference. The five steps were: 1 . General Needs; 2. Program Formulation; 3. Diagrams; 4. Circulation/Sectors/Volumes; and 5. Preliminary Study.

The second document was a structured form to be posted on online communities related to UFOP. The questions were aimed at making the program of the building clearer and more concise.

This form gave way to an open Google Spreadsheet with the actual program to be followed. It contained three main subjects: internal spaces; accessories; and project guidelines. Columns with tentative areas, orientations, necessity for external connection and relationship to other spaces were added. A total area of 380 square meters was preliminarily agreed upon. Again, anything done in the previous steps could at this point be complemented or improved, resulting therefore in changes to the current phase of the process.

\section{From Program to Space}

The next step was, then, to decide where on the campus the building would be located. A few available spots were surveyed on foot. The preliminary program along with the relationship between the different spaces was turned into a diagram and roughly drawn on scale using Space Syntax, an add-on for Grasshopper. The diagram was then overlaid on aerial photography of the different available spots, with one particular plot of land being deemed adequate by the participants. The result is shown below.

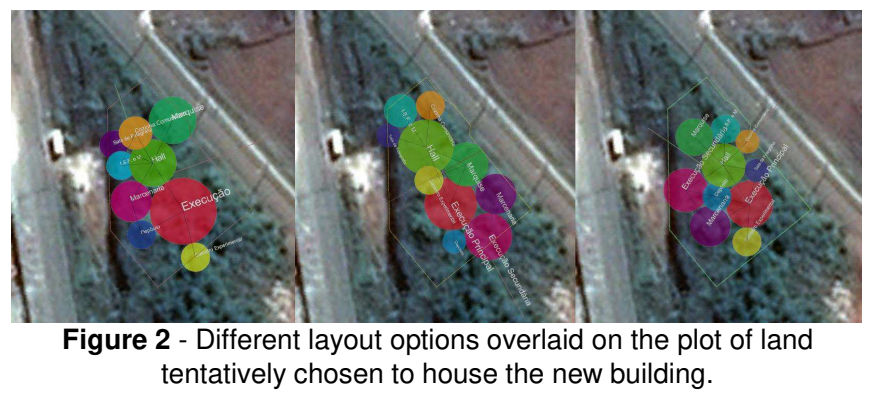

\section{Deciding on a design}

The last step was, then, to decide on a design for the diagrams that were proposed.

Following discussions between the workshop participants, it was agreed that each phase should observe four types of freedom inspired by the Free Software Definition and outlined by Fuller and Haque (2008):

"* The freedom to run the program, for any purpose (freedom $0)$.

* The freedom to study how the program works, and adapt it to your needs (freedom 1). Access to the source code is a precondition for this.

* The freedom to redistribute copies so you can help your neighbor (freedom 2).

* The freedom to improve the program, and release your improvements to the public, so that the whole community benefits (freedom 3). Access to the source code is a precondition for this."

In our case, these freedoms were freely adapted as follows:

- $\quad$ The freedom to propose any new concept or idea;

- $\quad$ The freedom to get to know in detail any concept proposed by anyone else;

- The freedom to adapt and improve upon any design published at any time;

- $\quad$ The freedom (and responsibility) to publish any work done in this context so that anyone else can scrutinize and improve upon one's work.

A similar process to the one described on the poster phase was developed: each phase consisted of an open call for proposals; a second open call for improvement upon other people's proposals; and a voting phase on the postulated submissions. This happened three times: one for preliminary plans; one for preliminary sketches; and one for preliminary projects. Some of the results are shown below. 

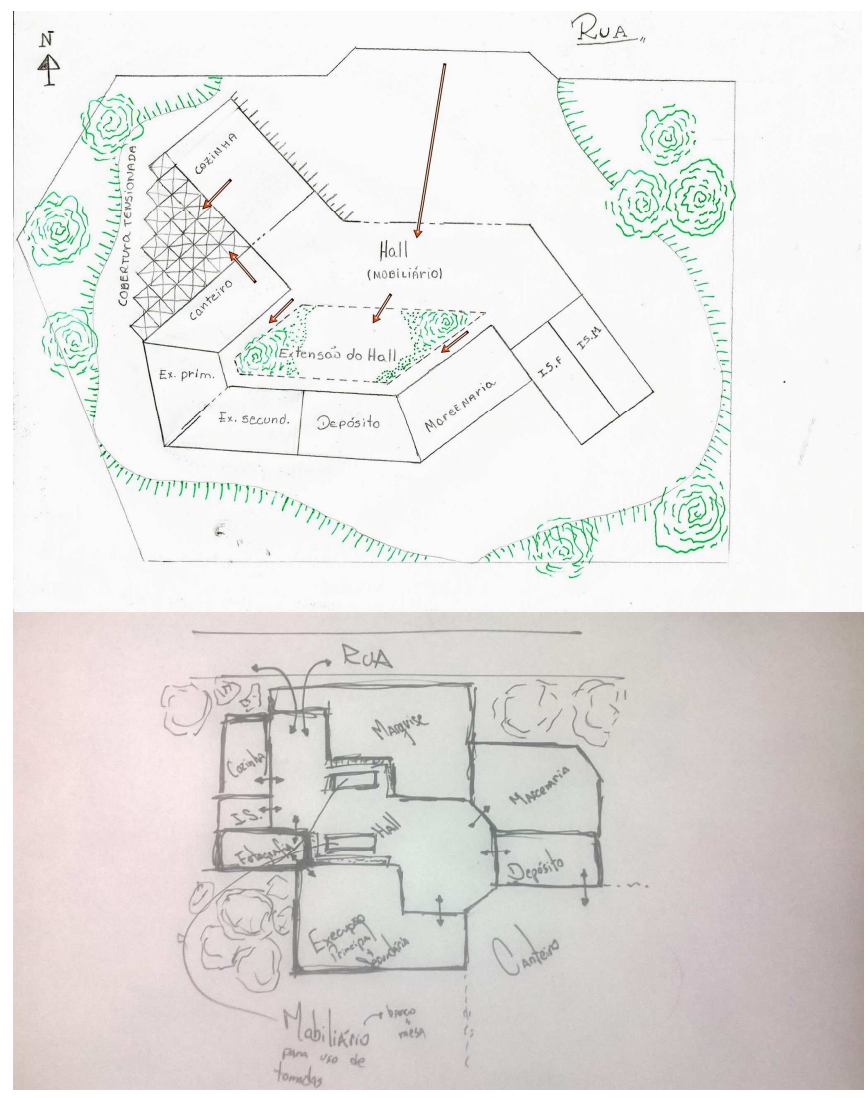

Figure 3 - Initial plan proposals.

At each phase, the following cycle took place:

1. Sketches are laid out;

2. Anyone is free to work or improve upon any other sketch;

3. Each finished sketch was presented to the community for suggestions;

4. A voting phase was held using Facebook. The results were not eliminatory, but seen merely as an indication of the community's preferences;

5. The process moved on to the next phase.

In this way, all ideas started out as individual creations, but soon blended with other ideas. The four levels of freedom mentioned above were observed throughout the process. Though not all participants had equal influence in the final design, the group concluded that each of the final designs changed enough over the process that they could not be linked back to any single initial proposal. In other words, every idea put forth in the beginning of the process changed enough that it became a collective creation in the end.

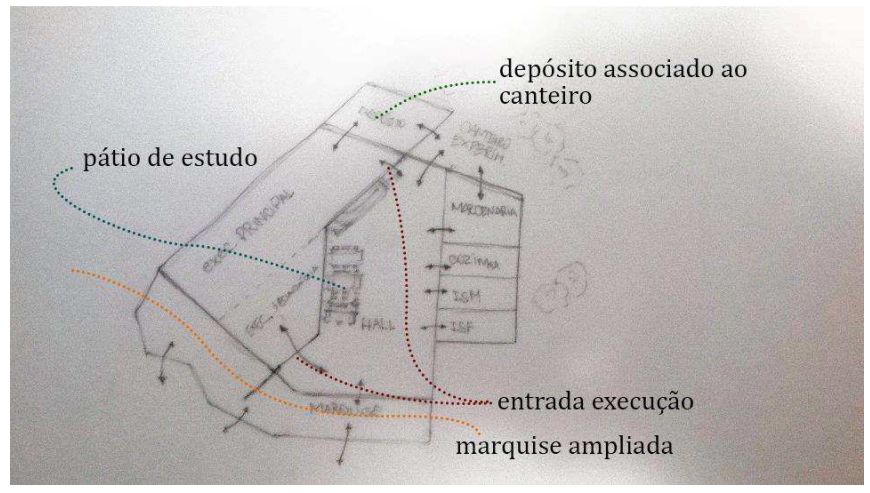

Figure 4 - One student's interactions over another's proposal.

As was expected due to the different levels of expertise possessed by each participant, many different tools were used to work upon each design iteration. Manual sketches, in the initial phases, and 3D models, in the final proposals, were predominant throughout the process. This stemmed from an objective intention of keeping the ideas easy to understand for people not familiar with technical architecture drawings, and could eventually be enhanced by the use of physical models.

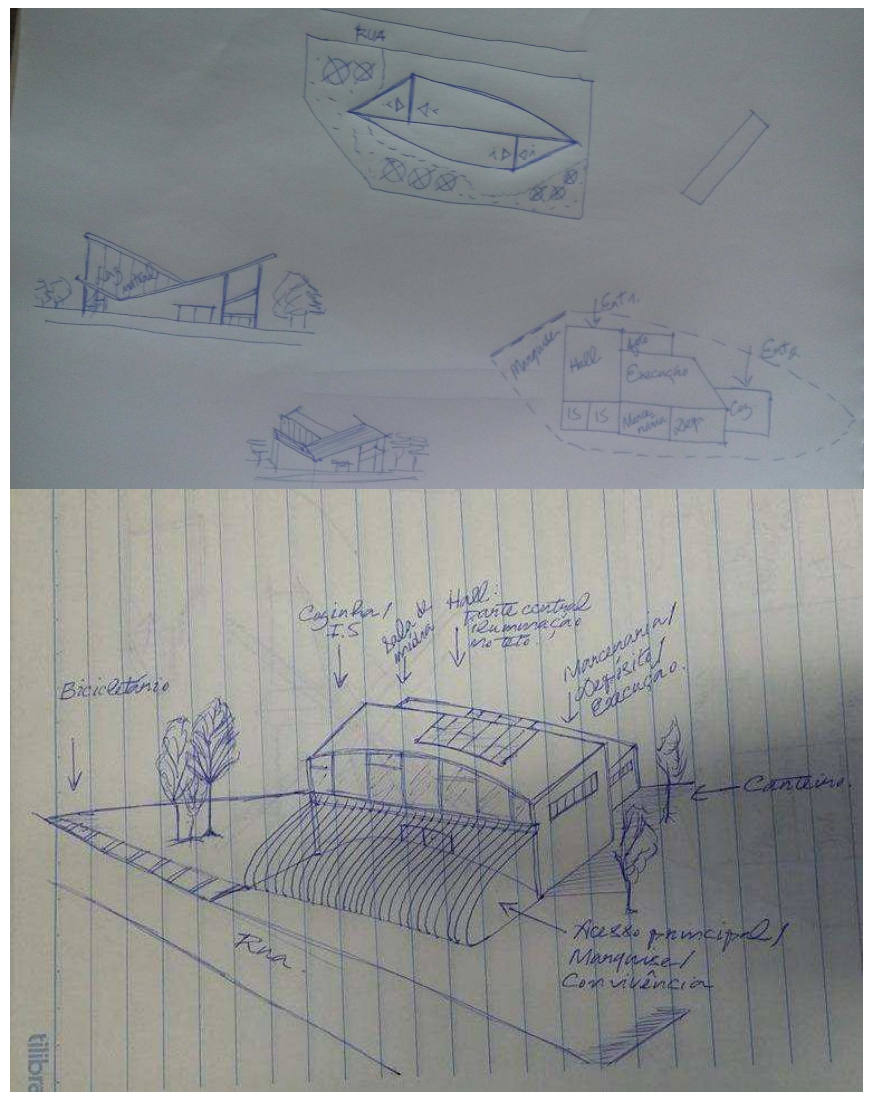

Figure 5 - Hand sketches over the proposed plans.

A final design was not agreed upon. It was proposed that the two leading alternatives (Figure 6) might be 3D-printed and displayed at the university campus for further involvement of 
the general community in the design process, which could be done when the university decided to finalize the design in order to build the proposed pavilion.
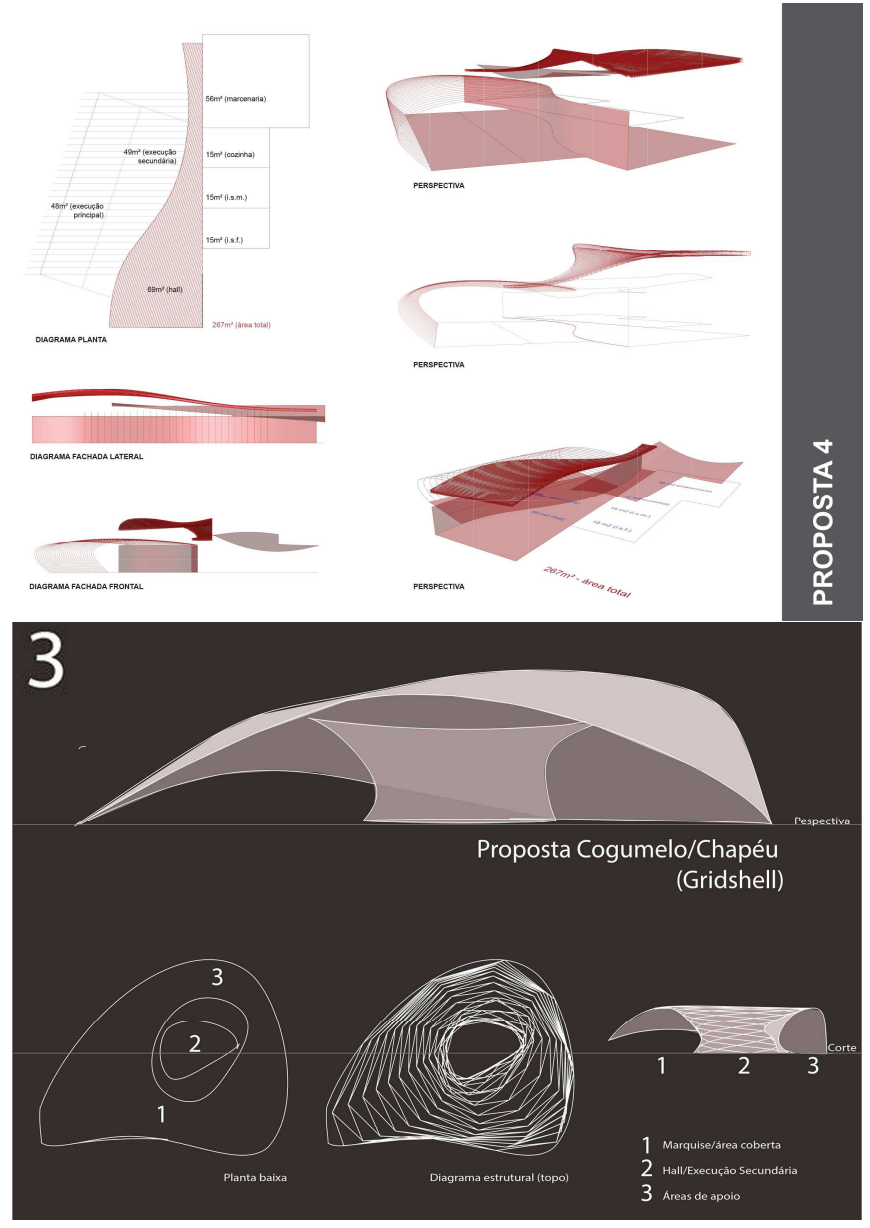

Figure 6 - Two of the final proposals developed using Grasshopper.

The same students later took part in another collaborative design experience that built upon the first one. Though the subject was much simpler, it was clear that, after the first experience, the students involved felt much more comfortable deciding on a program, negotiating different processes and, in this case, executing the object - a wooden set of pieces that fit the concrete benches at the university (Figure 7).

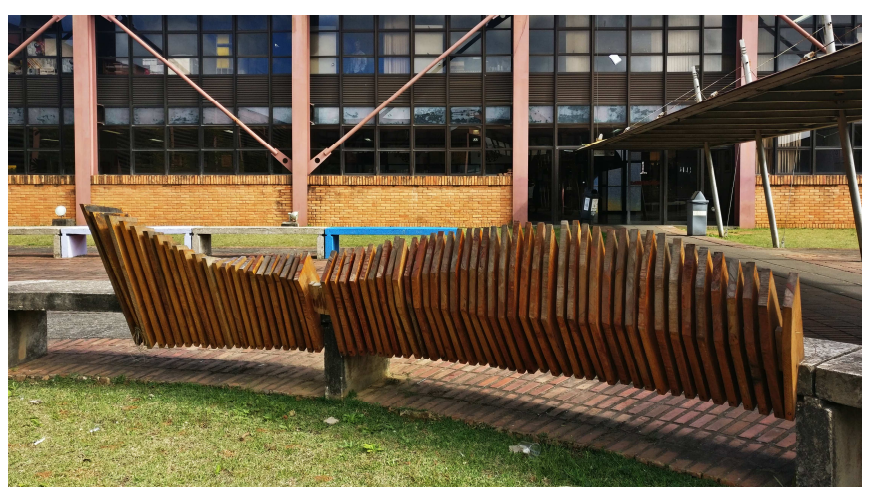

Figure 7 - The product of the second collaborative workshop. There was a consensus among the students that the first workshop constituted an important learning experience that made them more comfortable participating in a collaborative design process.

\section{Discussion}

Openness is not a binary attribute, but a spectrum (Broad, 2015). The same can be said of participation: a given process can be participatory to varying degrees. Even if participatory processes carry (rightfully) a positive bias in today's architectural body of knowledge, there is still much to be learnt about how different processes yield different types and levels of participation.

There is, furthermore, an inherent paradox in participatory processes, as discussed in the beginning of this paper. Processes that are not open enough can limit or induce participation and be used to legitimize decisions that are inherently centralized. On the other hand, too much openness can cause immobility. Throughout the exercise presented here, a delicate balance had to be constantly pursued between too much and too little openness.

Our most relevant finding was that this balance was not static. When the collaborative workshop began, participants had the expectation of a centralized teaching process, and this expectation generated what began, indeed, as a hierarchical relationship between teacher and students. With each new experience, students and members of the different bodies of people interested in the project expressed further willingness to collaborate in the process. At no point did the experience become entirely horizontal, nor was it expected to, since different people have different interests and capacities that motivate them to get involved in varying intensities and in different ways.

The principle that each phase of the process remain open to everyone (both for consultation and for interference) proved useful as the community felt more comfortable in advancing towards the open end of the participatory spectrum. The participation framework was initially conceived individually; but since it remained open and was systematically revisited with each new phase and iteration, it ended up as a collective product. 


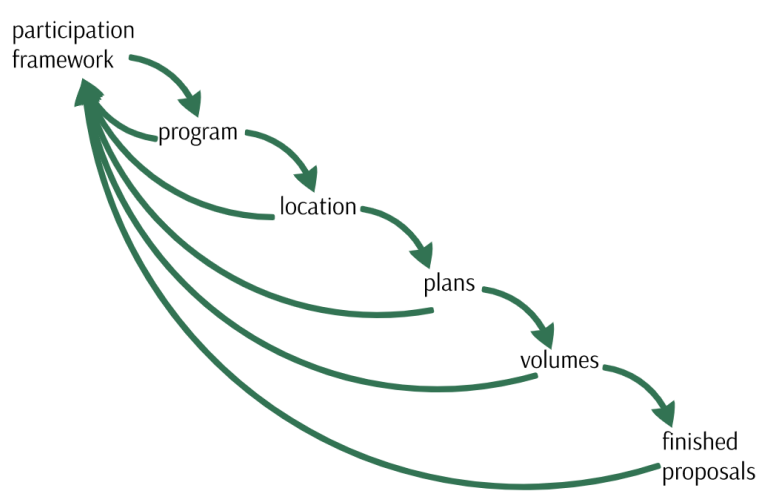

Figure 8 - A schematic layout of the cyclical process; as each new phase began the entire process was revisited, in a parametric fashion, with the initial phases becoming increasingly consolidated with each new iteration.

In closing, we ponder that, since the way each collaborative process is designed heavily influences its output, the process should, as much as possible, be designed collaboratively. This can be accomplished by promoting and sharing further collaborative experiences, in the spirit of the Creative Commons framework, so that others might test, criticize and build upon this branch of knowledge. Each new experience increases a person's awareness of the possibility of taking part in the process of shaping their environment, enabling them to participate more intensely in further processes.

A Wiki-like website themed around building and urban design processes could eventually be useful for gathering and systematizing experiences such as this one. In this way, each new collaborative design experience is, simultaneously, a design process in itself as well as an iteration that advances collaborative processes in general.

\section{Acknowledgments}

The nature of this experience means that there is not enough space here to credit everyone involved in it. The following students are worth mentioning for their contributions to the workshops: Adriely Pechutti, Cinthia Reis, Renata Ito, Renato Cruz and Tales Capute.

The Federal University of Ouro Preto (UFOP) deserves credit for hosting the workshops.

The Federal University of Minas Gerais (UFMG), through its NPGAU program, helped making this research possible.

\section{References}

Arnstein, S. R. (1969). A ladder of citizen participation. Journal of the American Institute of planners, 35(4), 216-224.

Ashby, W. R. (1964). An introduction to cybernetics: Methuen.

Banham, R. (1991). A black box: the secret profession of architecture. New Statesman and Society, 3, 22-25.

Broad, E. (2015). Closed, shared, open data: what's in a name? Retrieved from http://theodi.org/blog/closed-shared-open-datawhats-in-a-name

Friedmann, J. (1987). Planning in the Public Domain: From Knowledge to Action: Princeton University Press.

Fuller, M., \& Haque, U. (2008). Urban Versioning System 1.0 (Vol. 2): Lulu. com.

Kapp, S., \& Baltazar, A. P. (2012). The Paradox of Participation: A Case Study on Urban Planning in Favelas and a Plea for Autonomy. Bulletin of Latin American Research, 31(2), 160-173. doi: $10.2307 / 41410613$

Noveck, B. S. (2015). Smart citizens, smarter state: The technologies of expertise and the future of governing: Harvard University Press. 